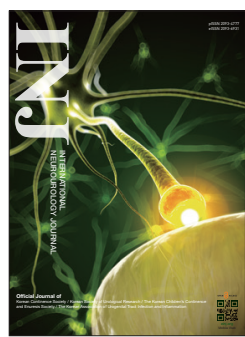

\title{
Current Pharmacologic Approaches in Painful Bladder Research: An Update
}

\author{
Karl-Erik Andersson ${ }^{1,2}$, Lori Birder ${ }^{3}$ \\ ${ }^{1}$ Institute for Regenerative Medicine, Wake Forest University School of Medicine, Winston Salem, NC, USA \\ ${ }^{2}$ Institute of Laboratory Medicine, Lund University, Lund, Sweden \\ ${ }^{3}$ Department of Medicine, University of Pittsburgh School of Medicine, Pittsburgh, PA, USA
}

The symptoms of interstitial cystitis (IC)/bladder pain syndrome (BPS) may have multiple causes and involve many contributing factors. Traditional treatments (intravesical instillations) have had a primary focus on the bladder as origin of symptoms without adequately considering the potential influence of other local (pelvic) or systemic factors. Systemic pharmacological treatments have had modest success. A contributing factor to the low efficacy is the lack of phenotyping the patients. Individualized treatment based on is desirable, but further phenotype categorization is needed. There seems to be general agreement that IC is a unique disease and that BPS is a syndrome with multiple pathophysiologies, but this has so far not been not been well reflected in preclinical research with the aim of finding new pharmacological treatments. Current research approaches, including anti-nerve growth factor treatment, anti-tumor necrosis factor- $\alpha$ treatment, activation of SHIP1 (AQX-1125), and P2X3 receptor antagonists, and $\alpha_{1}$-adrenoceptor antagonists are potential systemic treatments, implying that not only the bladder is exposed to the administered drug, which may be beneficial if the IC/BPS is a bladder manifestation of a systemic disease, or negative (adverse effects) if it is a local bladder condition. Local treatment approaches such as the antagonism of Toll-like receptors (which still is only experimental) and intravesical liposomes (with positive proof-of-concept), may have the advantages of a low number of systemic adverse effects, but cannot be expected to have effects on symptoms generated outside the bladder. Assessment of which of the treatment approaches discussed in this review that can be developed into useful therapies requires further studies.

Keywords: Nerve Growth Factor; Tumor Necrosis Factor-alpha; SHIP1; P2X3 Receptor; Toll-Like Receptors; Liposomes

- Conflict of Interest: No potential conflict of interest relevant to this article was reported.

\section{INTRODUCTION}

In the search of targets for pharmacologic treatment of interstitial cystitis (IC)/bladder pain syndrome (BPS), there has traditionally been a primary focus on the bladder and lower urinary tract as origin of symptoms without adequately considering the potential influence of other local (pelvic) or systemic factors.
Several epidemiological studies have shown that IC/BPS is commonly associated with other chronic pain conditions, including fibromyalgia, irritable bowel syndrome, and chronic fatigue syndrome, which suggests that IC/BPS may involve systemic pathophysiology, including changes in the central nervous system. Thus, it has to be decided whether to treat a local bladder disease or a systemic disease with bladder manifesta-
Corresponding author: Karl-Erik Andersson (iD https://orcid.org/0000-0003-0665-3486 Institute of Laboratory Medicine, Lund University, 22362 Lund, Sweden E-mail: karl-erik.andersson@med.lu.se / Tel: +46-705522722

Submitted: November 13, 2017 / Accepted after revision: November 26, 2017
(7) (5) This is an Open Access article distributed under the terms of the Creative Commons Attribution Non-Commercial License (http://creativecommons.org/licenses/by-nc/4.0/) which permits unrestricted non-commercial use, distribution, and reproduction in any medium, provided the original work is properly cited. 
tions. There may be multiple causes and contributing factors that can manifest in the symptoms of IC/BPS, and there may also be multiple patient subgroups or phenotypes. Recently some messages were delivered [1] that may give important directions for future research: (1) IC is a unique disease. (2) BPS is a syndrome, not a disease. (3) Patients should be phenotyped before treatment. Current research on possible targets for treatment IC/BPS, some of which are discussed in this review, reflects to some extent these messages.

\section{METHODS}

A search for literature concerning recent preclinical and clinical research on pharmacological targets relevant for treatment of IC/BPS was performed. The electronic databases PubMed, Google Scholar, and Scopus were used to identify relevant clinical and animal studies. Keywords were entered as medical subject headings (MeSH) or as text words. The Mesh terms were used in various combinations and usually included the terms chronic pelvic pain, bladder, afferent signalling, cystitis, IC/BPS, treatment, pharmacology. Search results were assessed for their overall relevance to this review.

\section{EMERGING TARGETS}

Pain during bladder filling is a perception and signalling from afferent nerves, particularly from the bladder, but also from other pelvic organs ("cross-talk"), can be expected to play an important role in its generation. It is therefore not surprising that most recent research is "bladder-centric," implying that it has a focus on possible targets within the bladder. However, systemic administration of a drug implies that not only bladder is exposed, which may be beneficial if the IC/BPS is a bladder manifestation of a systemic disease, or negative (adverse effects) if it is a local bladder condition.

\section{ANTI-NERVE GROWTH FACTOR TREATMENT}

Elevated levels of nerve growth factor (NGF) have been demonstrated in the bladder of various animal models of bladder inflammation, e.g., chemical, neurogenic, and immune-mediated [2]. In rats, acute intravesical administration of NGF produced sensitization of bladder afferents, resulting in increased frequency of micturition and reduced micturition threshold (as measured by cystometry). In patients with IC/BPS the bladder expression of NGF was found to be increased [3], and urine obtained from IC/BPS patients contained increased levels of the neurotrophin [4]. A recent meta-analysis reported that urinary NGF could be a useful biomarker for the differential diagnosis of IC/BPS and overactive bladder as well as a predictive biomarker to help guide treatments [5]. The results from preclinical and clinical studies would therefore suggest inhibition of the release or actions of NGF to be reasonable treatment approaches. In a phase II study [6], the effects of tanezumab, a monoclonal NGF neutralizing antibody, given intravenously, were studied in 64 IC/BPS patients (34 receiving tanezumab). At week 6 tanezumab produced a significant reduction from baseline in average daily pain score vs. placebo and a significantly higher proportion of patients on tanezumab responded as improved in the global response assessment. Tanezumab also significantly reduced urgency episode frequency vs. placebo, but had no significant effect on Interstitial Cystitis Symptom Index score, micturition frequency, or mean voided volume per micturition. Side effects included headache and paraesthesia. This study suggested a positive effect of the agent. However, serious adverse events were reported in an initial study of tanezumab in osteoarthritis, in which bone necrosis developed and total joint replacements were needed, and the agent was temporarily put on clinical hold [7]. After the clinical program was resumed in 2012, several studies on osteoarthritis have been performed, and in a recent meta-analysis tanezumab was shown to provide superior pain relief and improvement in physical function and patient's global assessment over placebo in knee and hip osteoarthritis patients, and tanezumab was generally well tolerated with acceptable adverse effects [8].

Fulranumab, another human monoclonal antibody directed against NGF, showed efficacy in patients with moderate-to-severe osteoarthritis pain [9]. Wang et al. [10] performed a multicenter, double-blind study on adult patients with IC/BPS. The study was terminated prematurely for the same reasons as tanezumab (see above), but 31 patients (of 70 patients targeted) were randomized, 17 to placebo and 14 to fulranumab, with 15 and 10 patients, respectively, receiving all 3 doses of doubleblind treatment. There was no statistically significant difference between treatment groups for the primary endpoint (average daily pain intensity score). However, the authors did not exclude the possibility that the drug would provide clinical benefit in a larger study and/or specific populations [10]. This may be the case, but even if fulranumab was well tolerated in this study, in the osteoarthritis study by Sanga et al. [9], rapid progression 
of osteoarthritis was observed as a safety signal.

NGF may be an important pathophysiological factor inducing pain-related symptom in IC/BPS. However, even if the results with tanezumab may provide proof-of-concept evidence that inhibition of NGF can be effective for the treatment of IC/ BPS, potential safety issues related to systemic administration have to be clarified. This opens up for local approaches such as liposome-based intravesical therapy targeting NGF $[11,12]$.

\section{ANTI-TUMOR NECROSIS FACTOR- $\boldsymbol{\alpha}$ TREATMENT}

The tumor necrosis factor (TNF) is a proinflammatory cytokine released by immune cells which may be involved in acute and chronic inflammation in autoimmune diseases [13]. The TNF and TNF receptor superfamilies have a wide range of actions including promotion of cellular differentiation, survival, and production of inflammatory cytokines and chemokines $[14,15]$. Interestingly, Ogawa et al. [16] investigating genes responsible for ulcerative IC, found an increased mRNA expression of several genes related to immune and inflammatory responses in bladder urothelium of patients with ulcerative IC, including TNFSF14 (TNF ligand superfamily member 14), a lymphotoxin-like inducible protein that has an important role in the pathogenesis of autoimmune disease. Such changes were not found in patients with symptoms but without ulcerations.

Clinically, the anti-inflammatory effect of TNF blockade has been explored for the treatment various diseases $[14,15]$. Among several TNF antagonists, adalimumab, a fully human high-affinity, recombinant immunoglobulin $\mathrm{G}_{1}$ anti-TNF monoclonal antibody [13], has been tested in IC/BPS patients [17]. Adalimumab has a high selectivity and affinity for TNF and reduces TNF-induced inflammatory responses.

Bosch [17] reported the results of a phase III, randomized, double-blind, placebo-controlled proof-of-concept study on the effects of adalimumab in 43 patients (21 receiving drug) with moderate to severe IC/BPS. The patients were given $80-\mathrm{mg}$ subcutaneous adalimumab followed by $40 \mathrm{mg}$ every 2 weeks or subcutaneous placebo for 12 weeks. Compared to baseline, adalimumab treatment resulted in a statistically significant improvement in outcome measures (O'Leary-Sant Interstitial Cystitis Symptom and Problem Indexes, Interstitial Cystitis Symptom Index, Interstitial Cystitis Problem Index, and Pelvic Pain, Urgency, Frequency Symptom Scale). After 12 weeks of treatment, 11 of 21 patients (53\%) in the adalimumab group had a
$50 \%$ or greater improvement in global response assessment. There were no significant adverse events. However, the drug failed to demonstrate positive proof-of-concept compared to placebo due to a significant placebo effect.

Anti-TNF therapeutics have been successful and changed the management of autoimmune diseases such as rheumatoid arthritis [18]. However, so far it is unclear if this drug principle can be developed to a useful treatment alternative for patients with IC/PBS, and further controlled studies are needed.

\section{ANTAGONISTS OF TOLL-LIKE RECEPTORS}

It is well established that there is an overlap between IC/BPS and known autoimmune disorders, such as Sjoegren syndrome, systemic lupus erythematosus (SLE), rheumatoid arthritis, and ulcerative colitis [19-21]. Toll-like receptors (TLRs) play a key role in the innate immune system and in biological defense mechanisms against external pathogens such as bacteria, fungus, and virus [22]. Among the TLRs demonstrated in humans, TLR7 is expressed not only in immune reactive cells but also neurons and glia cells. TLR7 is associated with the development and maintenance of inflammation and pain and has been considered to contribute to the development of several autoimmune diseases, such as SLE and Sjoegren syndrome [23,24].

Ichihara et al. [25] studied TLR7 expression in bladder biopsy specimens from patients with Hunner-type IC. They also explored the functional roles of TLR7 in bladder inflammation and nociception in mice. Ichihara et al. [25] found that the number of TLR7 immuno-reactive cells and the mRNA expression of TLR7 were significantly higher in IC specimens than in samples from controls without the disease. Loxoribine, a TLR7 agonist, instilled in the bladder of C57BL/6N female mice, induced edema, congestion, and inflammation, and significantly increased the TLR7-mRNA expression. The drug also significantly increased licking behaviour, voiding frequency, and afferent nerve activities associated with decreased single-voided volume and intercontraction interval of micturition. In addition, activation of TLR7 induced cystitis with sensory hyperactivity. Hydroxychloroquine, a TLR7 antagonist, instilled intravesically, reversed the loxoribine induced cystometric and voiding behavioural changes. It may be speculated if blocking the TLR7 pathway in the bladder of IC patients can be a new treatment approach. Further studies are needed to confirm and explore the translational impact of these observations. 


\section{AQX-1125, A MODULATOR OF IMMUNE/ INFLAMMATORY PROCESSES}

AQX-1125 represents a new pharmaceutical class of compounds [26], activators of SH2-containing inositol-5' -phosphatase 1 (SHIP1). SHIP1 is an intracellular protein whose expression is primarily restricted to cells of the haematopoietic lineage. It is a negative regulator of the PI3K pathway and suppresses intracellular signalling by catalysing the degradation of PIP3 to $\mathrm{PI}(3,4)$ P2 (PIP2), thus suppressing PIP3-mediated cellular effects. In addition to its catalytic function, SHIP1 can also act as a negative regulator of signalling through its interactions with other proteins [27]. SHIP1 is a negative regulator of many inflammatory signalling processes of the immune system, and has documented roles in controlling vital cell functions such as development, proliferation, activation, cytokine secretion and migration [28]. Activation of SHIP1 has an anti-inflammatory effect [29]. The highly restricted expression pattern of SHIP1 in addition to its ability to act as a negative regulator of immune cell signalling makes it a highly attractive target for the development of anti-inflammatory therapeutics, and pharmacological activation of SHIP1 has emerged as a novel approach for the therapy of various inflammatory diseases [26,29,30].

AQX-1125 has been studied in a short-term, phase II, randomized, placebo controlled study, which included patients with moderate to severe IC/BPS, some with Hunner lesions (Aquinox Trial) [31]. Thirty-seven IC/BPS women were treated with the compound and 32 with placebo. Women treated with AQX-1125 showed a significant reduction in bladder pain and improvement of symptoms at 6 weeks compared to placebotreated women [31]. Thus, anti-inflammatory treatment targeting the PI3K pathway could potentially be a useful strategy for the treatment of IC/BPS patients, especially when bladder inflammatory changes such as Hunner lesions are identified in the bladder [16].

\section{P2X3 RECEPTOR ANTAGONISTS}

Bladder distension stimulates the urothelium to release factors, such as ATP, from its mucosal and serosal surfaces [32]. ATP then activates $\mathrm{P} 2 \mathrm{X} 3$ receptors in bladder afferents to modulate bladder activity as evidenced by experimental studies of P2X3 knockout mice [33]. In IC/BPS, the urothelial expression of $\mathrm{P} 2 \mathrm{X} 3$ is increased and the stimulatory ATP mechanism is upregulated [34-37]. It seems therefore logical to expect that inhi- bition of ATP release or blockade of $\mathrm{P} 2 \mathrm{X} 3$ receptors would be effective ways of blocking afferent activity involved in symptom generation.

AF-219 is an orally active small molecule antagonist at human P2X3-containing receptors that in a double-blind, placebo-controlled, 2-period, crossover study was effective as an antitussive [38]. Moldwin et al. [39] performed a placebo-controlled clinical trial with AF-219 in female IC/BPS patients, 36 treated with the drug and 38 with placebo. Patients treated with AF-219 for 4 weeks had improvement in the key symptoms of IC/BPS such as pain scores, urinary urgency and in global response assessment, compared to placebo-treated patients. There were 5 patients ( 4 in the AF-219 arm and 1 in the placebo arm) with Hunner lesions on cystoscopy. Thus, targeting the ATP and $\mathrm{P} 2 \mathrm{X}$ receptor mechanism in the bladder may be a promising strategy for the treatment of IC/BPS with or without Hunner lesions.

\section{REDUCTION OF SYMPATHETIC OVERACTIVITY AND STRESS}

There is an increasing body of evidence suggesting that the sympathetic system is implicated in chronic painful conditions [40,41]. Studies have shown that $\alpha$-adrenoceptors (ARs) increase pain in human disorders such as complex regional pain syndrome which exhibit a number of parallels to IC/BPS [4245]. IC/BPS patients excrete high levels of urinary catecholamines [46,47], and a pathophysiologic role for a generalized cardiovascular and vasomotor autonomic nervous system abnormality in IC/BPS patients has been suggested. In a preliminary study, Chelimsky et al. [48] evaluated and compared the structural integrity of the autonomic nervous system in IC/BPS and control subjects, but found no differences, except that IC/ BPS patients had higher baseline heart rate. In a follow-up study the same group compared IC/BPS patients with those suffering from another disorder, myofascial pelvic pain with pain in the pelvis unrelated to bladder state. A higher baseline heart rate was confirmed, and 6 of 36 IC/BPS patients (22\%) were shown to have an autonomic neuropathy.

A link between chronic pain and the sympathetic system may be concluded from several animal experiments [49-52]. Charrua et al. [47] injected phenylephrine subcutaneously daily for 14 days in female rats and found increases in visceral pain, spinal Fos expression, bladder reflex activity, urinary spotting, and the number of expelled fecal pellets. The mucosa showed 
urothelial thinning and increased immunoreactivity for caspase 3 and bax. Trypan blue staining suggesting increased urothelial permeability was only observed in phenylephrine treated animals, which also showed increased sympathetic nerve density and urinary noradrenaline levels. Based on their results, Charrua et al. [47] concluded that excessive adrenergic stimulation of the bladder may contribute to the pathophysiological mechanisms of BPS/IC. In a follow-up study, Matos et al. [53] showed that phenylephrine-induced morphological bladder changes, bladder overactivity, and L6 spinal cord Fos expression, were reversed by the highly selective $\alpha_{1 \mathrm{~A}}$-AR antagonist, silodosin, but not by naftopidil, a relatively selective antagonist for $a_{1 D^{-}}$ ARs. Prazosin (which blocks all subtypes of $\alpha_{1}$-ARs) had an intermediate effect. Pain elicited by phenylephrine could be elicited in wild-type, but not TRPV1 knockout, mice. Silodosin also reversed the enhanced contraction responses to capsaicin found phenylephrine-treated rats. Primary afferents can coexpress $\alpha$-ARs and TRPV1 receptors [47,53], which may lead to interactions between sympathetic efferent nerves and nociceptive primary afferent nerves via a chemical cross-talk. When human urothelial cells (which express ARs) were treated with phenylephrine, ATP release, by mechanical stimulation or by lowering the thermal threshold of urothelial TRPV1, was enhanced. The authors suggested that activation of peripheral $\alpha_{1 A^{-}}$ ARs induces chronic visceral pain, probably through its interaction with TRPV1 and ATP release.

Chronic emotional stress may produce sensitization and enhanced excitability of afferent pathways innervating the lower urinary tract, and this is believed to play a role in the exacerbation and possibly the development of functional lower urinary tract disorders $[54,55]$. The water avoidance stress (WAS) in rodents reproduces signs of nociception and bladder changes seen in IC/BPS patients [56,57]. Matos et al. [57] explored the possible role of a1A-ARs in bladder pain and morphological changes induced by WAS in a group of female Wistar rats. A separate WAS group received silodosin daily. Lower abdominal pain was determined by performing sensitivity to Von Frey filaments, and bladder reflex activity was assessed by cystometry in anaesthetised animals. WAS increased urinary noradrenaline levels and bladder frequency was increased in WAS animals, and the mechanical pain threshold was decreased. These effects were reversed by silodosin. Morphological changes such as lymphocytic and mast cells infiltration in the mucosa and mild urothelial disruption seen in the WAS group were absent in WAS+silodosin group.
These results suggest that in rats $\alpha_{1 \mathrm{~A}}$-ARs have an important role in the appearance of bladder pain induced either by pharmacological stimulation or by WAS, and that $\alpha_{1 \mathrm{~A}}-\mathrm{AR}$ inhibition can prevent not only pain but underlying morphological changes. It is not surprising that $\alpha_{1}$-AR agonist induced changes may be reversed by antagonists. However, WAS induced functional and morphological changes in the bladder probably have a more complex pathophysiology, and chronic stress can in addition to sympathetic overactivity, involve activation of the hypothalamic-pituitary axis, dysregulation of the serotonergic pathways, and central sensitization [55]. Nevertheless, the finding that selective $\alpha_{1 \mathrm{~A}}$-AR inhibition can improve both functional and morphological changes in WAS animals, is of potential translational significance. Since BPS/IC patients present high blood levels of noradrenaline [46,47], $\alpha_{1 \mathrm{~A}}$-AR stimulation may be an additional trigger for bladder dysfunction in these patients. The response to treatment with $\alpha_{1 \mathrm{~A}}-\mathrm{AR}$ antagonists in patients with IC/BPS would be worthwhile exploring.

\section{INTRAVESICAL LIPOSOMES}

Liposomes are lipid vesicles composed of phospholipid bilayers surrounding an aqueous core [58]. The lipidic bilayer structure of liposomes facilitates their adherence to the apical membrane surface of luminal cells in the bladder. Liposomes are taken up by urothelial cells through clathrin-mediated endocytosis, and their vesicular shape allows them to use the endocytosis uptake machinery after instillation [12]. Empty liposomes can protect damaged urothelium and have shown therapeutic benefits for IC/BPS patients [12]. In addition, liposomes can carry various drugs to penetrate urothelium and modulate afferent neurotransmission [12]. The mechanism of action for empty liposomes in the bladder was suggested to involve a combination of a physical coating on the urothelium, and a drug-like action that promotes repair and regeneration of the bladder lining [12]. The primary constituent current used for instillation of liposome into bladders of IC patients is sphingomyelin, which has an anti-inflammatory effect.

The first clinical study was performed by Chuang et al. [59] who compared the response of intravesical liposome therapy to that of oral pentosan polysulfate. Twenty-four patients with moderate IC/BPS were prospectively included in the study, and 12 of them were given $80 \mathrm{mg}$ of liposomal therapy instilled once weekly for 4 weeks. The other 12 patients received $300 \mathrm{mg}$ of oral pentosan polysulfate daily. Outcomes were measured 
using the O'Leary Sant score, pain assessment scale and global response assessment at 4 and 8 weeks posttreatment. Statistically significant decreases in pain, urgency and the O'Leary-Sant symptom score were noted in the liposome group at 4 weeks, which remained at 8 weeks. Urinary frequency and nocturia scores were comparable to the pentosan polysulfate group. There were no side effects, which were confirmed in a later study including 17 patients [60]. Peters et al. [61] followed 14 IC/BPS patients (11 women, 3 men) who underwent weekly instillations of liposomes for 4 weeks. Efficacy measurements included pain visual analog scales, voiding diaries, global response assessments, and O'Leary-Sant Interstitial Cystitis Symptom and Problem Indices. Cystoscopic improvements were noted in three patients, no change in 10 and worsening in 1 patient. No adverse side effects were noted and outcomes were positive with improvements in pain, urgency, and overall symptoms scores. Improvements in urgency were sustained at 8 weeks. Although pain scores significantly improved at 4 weeks, they were found to be back to baseline at 8 weeks.

Intravesical liposome therapy, both with empty liposomes but particularly with liposomes carrying active drugs, seems to be a promising approach for treatment of IC/BPS patients. However, effectiveness has to be assessed in large randomized controlled trials.

\section{SUMMARY AND CONCLUSIONS}

Inflammation and signalling from afferent nerves in the bladder and other pelvic organs, can be expected to play an important role in the symptoms of IC/BPS. Current research approaches including anti-NGF treatment, anti-TNF- $\alpha$ treatment, activation of SHIP1 (AQX-1125), P2X3 receptor antagonists, and $\alpha_{1}$-AR antagonists, despite having bladder-centric rationale, are potential systemic treatments which implies that they could be expected to have effects both on the bladder and on nonbladder manifestations of IC/BPS. Local treatment approaches such as the antagonism of TLRs (which still is only experimental) and intravesical liposomes (with positive proof-of-concept), may have the advantages of a low number of systemic adverse effects, but cannot be expected to have effects on symptoms generated outside the bladder. Individualized treatment based on phenotype (IC is a separate disease, BPS a syndrome) seems rational. However, assessment of which of the treatment approaches discussed that can be developed into useful therapies requires further studies.

\section{REFERENCES}

1. Payne CK. The Empereror Has No Clothes. Lecture AUA, 2017 [Internet]. Berkeley (CA): UroToday; c2002-2017 [cited 2007 Nov 27] Available from: https://www.urotoday.com/video-lectures/bpsic/video/788-embedded-media2017-07-05-16-32-47.html

2. Ochodnicky P, Cruz CD, Yoshimura N, Cruz F. Neurotrophins as regulators of urinary bladder function. Nat Rev Urol 2012;9:62837.

3. Lowe EM, Anand P, Terenghi G, Williams-Chestnut RE, Sinicropi DV, Osborne JL. Increased nerve growth factor levels in the urinary bladder of women with idiopathic sensory urgency and interstitial cystitis. Br J Urol 1997;79:572-7.

4. Okragly AJ, Niles AL, Saban R, Schmidt D, Hoffman RL, Warner TF, et al. Elevated tryptase, nerve growth factor, neurotrophin -3 and glial cell line-derived neurotrophic factor levels in the urine of interstitial cystitis and bladder cancer patients. J Urol 1999;161:438-41.

5. Qu HC, Zhang W, Yan S, Liu YL, Wang P. Urinary nerve growth factor could be a biomarker for interstitial cystitis/painful bladder syndrome: a meta-analysis. PLoS One 2014;9:e106321.

6. Evans RJ, Moldwin RM, Cossons N, Darekar A, Mills IW, Scholfield D. Proof of concept trial of tanezumab for the treatment of symptoms associated with interstitial cystitis. J Urol 2011;185:171621.

7. Brown MT, Murphy FT, Radin DM, Davignon I, Smith MD, West CR. Tanezumab reduces osteoarthritic knee pain: results of a randomized, double-blind, placebo-controlled phase III trial. J Pain 2012;13:790-8.

8. Chen J, Li J, Li R, Wang H, Yang J, Xu J, et al. Efficacy and safety of tanezumab on osteoarthritis knee and hip pains: a meta-analysis of randomized controlled trials. Pain Med 2017;18:374-85.

9. Sanga P, Katz N, Polverejan E, Wang S, Kelly KM, Haeussler J, et al. Long-term safety and efficacy of fulranumab in patients with moderate-to-severe osteoarthritis pain: a phase ii randomized, doubleblind, placebo-controlled extension study. Arthritis Rheumatol 2017;69:763-73.

10. Wang H, Russell LJ, Kelly KM, Wang S, Thipphawong J. Fulranum$\mathrm{ab}$ in patients with interstitial cystitis/bladder pain syndrome: observations from a randomized, double-blind, placebo-controlled study. BMC Urol 2017;17:2.

11. Majima T, Tyagi P, Dogishi K, Kashyap M, Funahashi Y, Gotoh M, et al. Effect of intravesical liposome-based nerve growth factor antisense therapy on bladder overactivity and nociception in a rat model of cystitis induced by hydrogen peroxide. Hum Gene Ther 2017;28:598-609. 
12. Tyagi P, Kashyap M, Majima T, Kawamorita N, Yoshizawa T, Yoshimura N. Intravesical liposome therapy for interstitial cystitis. Int J Urol 2017;24:262-71.

13. Tracey D, Klareskog L, Sasso EH, Salfeld JG, Tak PP. Tumor necrosis factor antagonist mechanisms of action: a comprehensive review. Pharmacol Ther 2008;117:244-79.

14. Croft M, Duan W, Choi H, Eun SY, Madireddi S, Mehta A. TNF superfamily in inflammatory disease: translating basic insights. Trends Immunol 2012;33:144-52.

15. Croft M, Benedict CA, Ware CF. Clinical targeting of the TNF and TNFR superfamilies. Nat Rev Drug Discov 2013;12:147-68.

16. Ogawa T, Homma T, Igawa Y, Seki S, Ishizuka O, Imamura T, et al. CXCR3 binding chemokine and TNFSF14 over expression in bladder urothelium of patients with ulcerative interstitial cystitis. J Urol 2010;183:1206-12.

17. Bosch PC. Examination of the significant placebo effect in the treatment of interstitial cystitis/bladder pain syndrome. Urology 2014;84:321-6.

18. Li P, Zheng Y, Chen X. Drugs for autoimmune inflammatory diseases: from small molecule compounds to anti-TNF biologics. Front Pharmacol 2017;8:460.

19. van de Merwe JP. Interstitial cystitis and systemic autoimmune diseases. Nat Clin Pract Urol 2007;4:484-91.

20. van de Merwe JP, Nordling J, Bouchelouche P, Bouchelouche K, Cervigni M, Daha LK, et al. Diagnostic criteria, classification, and nomenclature for painful bladder syndrome/interstitial cystitis: an ESSIC proposal. Eur Urol 2008;53:60-7.

21. Homma Y, Ueda T, Tomoe H, Lin AT, Kuo HC, Lee MH, et al. Clinical guidelines for interstitial cystitis and hypersensitive bladder updated in 2015. Int J Urol 2016;23:542-9.

22. O’Neill LA, Golenbock D, Bowie AG. The history of Toll-like receptors - redefining innate immunity. Nat Rev Immunol 2013; 13:453-60.

23. Liu T, Gao YJ, Ji RR. Emerging role of Toll-like receptors in the control of pain and itch. Neurosci Bull 2012;28:131-44.

24. Nicotra L, Loram LC, Watkins LR, Hutchinson MR. Toll-like receptors in chronic pain. Exp Neurol 2012;234:316-29.

25. Ichihara K, Aizawa N, Akiyama Y, Kamei J, Masumori N, Andersson $\mathrm{KE}$, et al. Toll-like receptor 7 is overexpressed in the bladder of Hunner-type interstitial cystitis, and its activation in the mouse bladder can induce cystitis and bladder pain. Pain 2017;158:153845.

26. Stenton GR, Mackenzie LF, Tam P, Cross JL, Harwig C, Raymond J, et al. Characterization of AQX-1125, a small-molecule SHIP1 activator: Part 1. Effects on inflammatory cell activation and chemo- taxis in vitro and pharmacokinetic characterization in vivo. Br J Pharmacol 2013;168:1506-18.

27. Condé C, Gloire G, Piette J. Enzymatic and non-enzymatic activities of SHIP-1 in signal transduction and cancer. Biochem Pharmacol 2011;82:1320-34.

28. Kerr WG. Inhibitor and activator: dual functions for SHIP in immunity and cancer. Ann N Y Acad Sci 2011;1217:1-17.

29. Stenton GR, Mackenzie LF, Tam P, Cross JL, Harwig C, Raymond J, et al. Characterization of AQX-1125, a small-molecule SHIP1 activator: Part 2. Efficacy studies in allergic and pulmonary inflammation models in vivo. Br J Pharmacol 2013;168:1519-29.

30. Leaker BR, Barnes PJ, O’Connor BJ, Ali FY, Tam P, Neville J, et al. The effects of the novel SHIP1 activator AQX-1125 on allergen-induced responses in mild-to-moderate asthma. Clin Exp Allergy 2014;44:1146-53.

31. Nickel JC, Egerdie B, Davis E, Evans R, Mackenzie L, Shrewsbury SB. A phase II study of the efficacy and safety of the novel oral SHIP1 activator AQX-1125 in subjects with moderate to severe interstitial cystitis/bladder pain syndrome. J Urol 2016;196:747-54.

32. Wang EC, Lee JM, Ruiz WG, Balestreire EM, von Bodungen M, Barrick S, et al. ATP and purinergic receptor-dependent membrane traffic in bladder umbrella cells. J Clin Invest 2005;115:241222.

33. Cockayne DA, Hamilton SG, Zhu QM, Dunn PM, Zhong Y, Novakovic $S$, et al. Urinary bladder hyporeflexia and reduced pain-related behaviour in P2X3-deficient mice. Nature 2000;407:1011-5.

34. Sun Y, Chai TC. Effects of dimethyl sulphoxide and heparin on stretch-activated ATP release by bladder urothelial cells from patients with interstitial cystitis. BJU Int 2002;90:381-5.

35. Sun Y, Keay S, De Deyne PG, Chai TC. Augmented stretch activated adenosine triphosphate release from bladder uroepithelial cells in patients with interstitial cystitis. J Urol 2001;166:1951-6.

36. Sun Y, Chai TC. Up-regulation of $\mathrm{P} 2 \mathrm{X} 3$ receptor during stretch of bladder urothelial cells from patients with interstitial cystitis. J Urol 2004;171:448-52.

37. Sun Y, Chai TC. Augmented extracellular ATP signaling in bladder urothelial cells from patients with interstitial cystitis. Am J Physiol Cell Physiol 2006;290:C27-34.

38. Abdulqawi R, Dockry R, Holt K, Layton G, McCarthy BG, Ford AP, et al. P2X3 receptor antagonist (AF-219) in refractory chronic cough: a randomised, double-blind, placebo-controlled phase 2 study. Lancet 2015;385:1198-205.

39. Moldwin R, Kitt M, Mangel J, Beyer R, Hanno P, Butera P, et al. A phase 2 study in women with interstitial cystitis/bladder pain syndrome (IC/BPS) of the novel P2X3 antagonist AF-219 [abstract No. 
23]. In: 45th International Continence Society (ICS) Annual Meeting; 2015 Oct 6-9; Montreal, Canada. Bristol (United Kingdom): International Continence Society; 2015.

40. Mailis-Gagnon A, Bennett GJ. Abnormal contralateral pain responses from an intradermal injection of phenylephrine in a subset of patients with complex regional pain syndrome (CRPS). Pain 2004;111:378-84.

41. Chalaye P, Goffaux P, Bourgault P, Lafrenaye S, Devroede G, Watier A, et al. Comparing pain modulation and autonomic responses in fibromyalgia and irritable bowel syndrome patients. Clin J Pain 2012;28:519-26.

42. Drummond PD, Finch PM, Smythe GA. Reflex sympathetic dystrophy: the significance of differing plasma catecholamine concentrations in affected and unaffected limbs. Brain 1991;114(Pt 5): 2025-36.

43. Galloway NT, Gabale DR, Irwin PP. Interstitial cystitis or reflex sympathetic dystrophy of the bladder? Semin Urol 1991;9:148-53.

44. Torebjörk E, Wahren L, Wallin G, Hallin R, Koltzenburg M. Noradrenaline-evoked pain in neuralgia. Pain 1995;63:11-20.

45. Chémali KR, Gorodeski R, Chelimsky TC. Alpha-adrenergic supersensitivity of the sudomotor nerve in complex regional pain syndrome. Ann Neurol 2001;49:453-9.

46. Stein PC, Torri A, Parsons CL. Elevated urinary norepinephrine in interstitial cystitis. Urology 1999;53:1140-3.

47. Charrua A, Pinto R, Taylor A, Canelas A, Ribeiro-da-Silva A, Cruz $\mathrm{CD}$, et al. Can the adrenergic system be implicated in the pathophysiology of bladder pain syndrome/interstitial cystitis? A clinical and experimental study. Neurourol Urodyn 2015;34:489-96.

48. Chelimsky G, McCabe NP, Janata J, Elston R, Zhang L, Ialacci S, et al. Autonomic testing of women with interstitial cystitis/bladder pain syndrome. Clin Auton Res 2014;24:161-6.

49. Shir Y, Seltzer Z. Effects of sympathectomy in a model of causalgiform pain produced by partial sciatic nerve injury in rats. Pain 1991;45:309-20.

50. Chung K, Yoon YW, Chung JM. Sprouting sympathetic fibers form synaptic varicosities in the dorsal root ganglion of the rat with neuropathic injury. Brain Res 1997;751:275-80.

51. Yen LD, Bennett GJ, Ribeiro-da-Silva A. Sympathetic sprouting and changes in nociceptive sensory innervation in the glabrous skin of the rat hind paw following partial peripheral nerve injury. J
Comp Neurol 2006;495:679-90.

52. Longo G, Osikowicz M, Ribeiro-da-Silva A. Sympathetic fiber sprouting in inflamed joints and adjacent skin contributes to painrelated behavior in arthritis. J Neurosci 2013;33:10066-74.

53. Matos R, Cordeiro JM, Coelho A, Ferreira S, Silva C, Igawa Y, et al. Bladder pain induced by prolonged peripheral alpha $1 \mathrm{~A}$ adrenoceptor stimulation involves the enhancement of transient receptor potential vanilloid 1 activity and an increase of urothelial adenosine triphosphate release. Acta Physiol (Oxf) 2016;218:265-75.

54. Gao Y, Zhang R, Chang HH, Rodríguez LV. The role of C-fibers in the development of chronic psychological stress induced enhanced bladder sensations and nociceptive responses: a multidisciplinary approach to the study of urologic chronic pelvic pain syndrome (MAPP) research network study. Neurourol Urodyn 2017 Aug 9 [Epub]. https://doi.org/10.1002/nau.23374.

55. Sanford MT, Rodriguez LV. The role of environmental stress on lower urinary tract symptoms. Curr Opin Urol 2017;27:268-73.

56. Wang Z, Chang HH, Gao Y, Zhang R, Guo Y, Holschneider DP, et al. Effects of water avoidance stress on peripheral and central responses during bladder filling in the rat: a multidisciplinary approach to the study of urologic chronic pelvic pain syndrome (MAPP) research network study. PLoS One 2017;12:e0182976.

57. Matos R, Serrão P, Rodriguez L, Birder LA, Cruz F, Charrua A. The water avoidance stress induces bladder pain due to a prolonged alpha1A adrenoceptor stimulation. Naunyn Schmiedebergs Arch Pharmacol 2017;390:839-44.

58. Cortesi R, Nastruzzi C. Liposomes, micelles and microemulsions as new delivery systems for cytotoxic alkaloids. Pharm Sci Technolo Today 1999;2:288-98.

59. Chuang YC, Lee WC, Lee WC, Chiang PH. Intravesical liposome versus oral pentosan polysulfate for interstitial cystitis/painful bladder syndrome. J Urol 2009;182:1393-400.

60. Lee WC, Chuang YC, Lee WC, Chiang PH. Safety and dose flexibility clinical evaluation of intravesical liposome in patients with interstitial cystitis or painful bladder syndrome. Kaohsiung J Med Sci 2011;27:437-40.

61. Peters KM, Hasenau D, Killinger KA, Chancellor MB, Anthony M, Kaufman J. Liposomal bladder instillations for IC/BPS: an open-label clinical evaluation. Int Urol Nephrol 2014;46:2291-5. 